\title{
Preface
}

\section{Left Atrial Appendage: What \\ Do We Know? What Do We Need? Where Are We Going?}

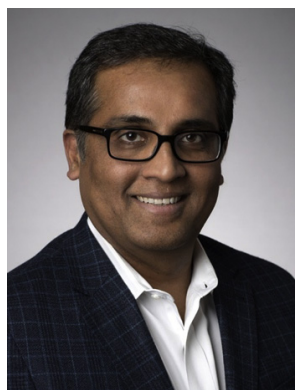

Dhanunjaya Lakkireddy, MD, FACC, FHRS

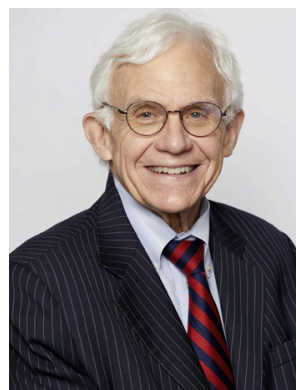

David R. Holmes Jr, MD, MACC Editors

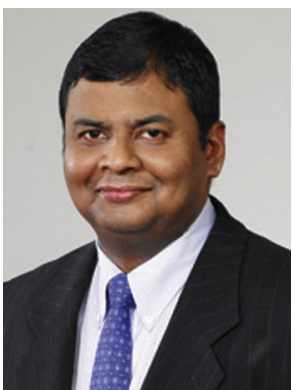

Saibal Kar, MD
Atrial fibrillation (AF) remains the focus of intense and increasing interest from multiple stakeholders, including family physicians, internists, general and interventional cardiologists, electrophysiologists, cardiovascular surgeons, and industry. This has been driven by the fact that it is the most common significant cardiac arrhythmia with an estimated $6,000,000$ patients by 2030 . Its clinical consequences include, among others, the need for bradycardia support pacemakers as well as tachycardia-induced cardiomyopathy and stroke.

Within the past decade, there has been emphasis on pulmonary vein isolation for treatment and prevention of AF; more recently, another area of major interest has centered on stroke prevention. For the latter indication, the closely interrelated triad of increasing $A F$, increasing patient age, and increasing incidence of stroke has been documented repeatedly.

This issue focuses on the prevention of stroke for which an increasing amount of information has become available. New opportunities continue to develop as we learn more about the pathophysiology of AF itself, the physiologic/anatomic basis of thrombus formation, the association of AF with systemic vascular disease, the increasing number of devices for left atrial exclusion, the patient, and anatomic characteristics in whom the risk-benefit ratio is optimal, the role of the left atrial appendage in the pathogenesis of AF, and its role in hemodynamics. Finally, we now have information on the unanticipated effects of device exclusion with the documentation of device-related thrombus and its optimal treatment as well as screening.

This multidisciplinary field remains robust; the unmet clinical needs remain substantial, and the scientific rigor being devoted to understanding the fundamental issues and problemsolving promises continued great advances in the future.

Dhanunjaya Lakkireddy, MD, FACC, FHRS The Kansas City Heart Rhythm Institution and Research Foundation HCA MIDWEST HEALTH Second Floor, $5100 \mathrm{~W}$ 110th St Overland Park, KS 66211, USA

David R. Holmes Jr, MD, MACC Department of Cardiovascular Diseases Mayo Clinic

200 First Street SouthWest Rochester, MN 55905, USA

Saibal Kar, MD

David Geffen School of Medicine at UCLA Structural Heart Disease Interventions \& Research Los Robles Regional Medical Center 227 W Janss Road, Suite 360 Thousand Oaks, CA 91360, USA

E-mail addresses: dlakkireddy@gmail.com (D. Lakkireddy) holmes.david@mayo.edu (D.R. Holmes) saibalkar60@gmail.com (S. Kar) 